BMJ Open

Diabetes

Research

\& Care

\section{Long-term metformin adherence in the Diabetes Prevention Program Outcomes Study}

\author{
Elizabeth Arquin Walker (10 , ${ }^{1}$ Jeffrey S Gonzalez, ${ }^{1,2}$ Mark T Tripputi, ${ }^{3}$ \\ Samuel Dagogo-Jack (D) , ${ }^{4}$ Margaret J Matulik, ${ }^{5}$ Maria G Montez, ${ }^{6}$ Sameh Tadros, ${ }^{7}$ \\ Sharon L Edelstein (1) , ${ }^{3}$ DPP Research Group
}

To cite: Walker EA, Gonzalez JS, Tripputi MT, et al. Long-term metformin adherence in the Diabetes Prevention Program Outcomes Study. BMJ Open Diab Res Care 2020;8:e001537. doi:10.1136/ bmjdrc-2020-001537

- Supplemental material is published online only. To view please visit the journal online (http://dx.doi.org/10.1136/ bmjdrc-2020-001537).

Received 7 May 2020 Revised 4 August 2020 Accepted 15 August 2020

Check for updates

C Author(s) (or their employer(s)) 2020. Re-use permitted under CC BY-NC. No commercial re-use. See rights and permissions. Published by BMJ.

For numbered affiliations see end of article.

Correspondence to Ms Sharon L Edelstein; dppmail@bsc.gwu.edu

\section{ABSTRACT}

Introduction To investigate long-term metformin adherence in the Diabetes Prevention Program Outcomes Study (DPPOS) by examining: (1) predictors of long-term adherence to study metformin and (2) whether metformin adherence was associated with incident type 2 diabetes. Research design and methods DPPOS was an open-label continuation of the randomized clinical trial (Diabetes Prevention Program (DPP)) in which eligible participants randomized to the metformin group were offered study metformin and followed over 11 years. A brief structured adherence interview was administered semiannually. Metformin adherence was assessed by pill counts. Predictors of metformin adherence were examined in multivariate regression models. Incident diabetes associated with metformin adherence and other variables was assessed in Cox proportional hazards models. Results Of 868 participants eligible to continue taking study metformin, 664 (76\%) took at least some metformin over 11 years, with 478 of them reporting problems with adherence. DPPOS cumulative adherence showed significant associations of higher adherence $(\geq 80 \%)$ with early adherence at 3 months in DPP $(p<0.001)$ and lower depression scores during DPPOS $(p<0.001)$; significant differences were also seen by race/ethnicity $(p<0.004)$. Predicting adherence by multivariate modeling showed odds of adherence significantly lower for Black participants and for participants reporting more than one barrier. Odds for adherence were significantly higher for those adherent early in DPP and those reporting at least one planned strategy to improve adherence. Higher metformin adherence was significantly associated with a lower diabetes risk $(p=0.04)$, even after adjustment for demographic variables, depression, and anxiety scores.

Conclusions In this long-term diabetes prevention study, early metformin adherence and planned strategies to promote adherence improved long-term adherence over 11 years; higher adherence to metformin was related to lower diabetes incidence. Incorporating strategies to promote adherence when initially prescribing metformin and counseling to support adherence over time are warranted.

\section{INTRODUCTION}

Individuals with an established disease are often presumed to have an intrinsic motivation to adhere to prescribed medications in

\section{Significance of this study}

What is already known about this subject?

- It is widely known that adherence to therapeutic medications is a challenge for the majority of individuals; gaps in knowledge exist regarding longterm adherence to preventive medications such as metformin.

\section{What are the new findings?}

- In this study, we report novel data from 11 years of the Diabetes Prevention Program Outcomes Study and its diverse participants related to: predictors of cumulative adherence; barriers to adherence and planned strategies to improve adherence; and a model of covariates for time to type 2 diabetes diagnosis. This medication adherence report includes both clinical and behavioral outcomes related to diabetes prevention.

\section{How might these results change the focus of} research or clinical practice?

- Predictors of long-term adherence to this preventive medication for type 2 diabetes include early adherence in the course of treatment, which supports interventions to promote adherence at initiation of metformin. In light of the challenges in the prevention or delay of type 2 diabetes, these findings offer evidence from a long-term study of diverse participants to support initiating strategies to promote adherence when metformin is first prescribed in clinical practice and continuing to provide brief counseling over long-term use of metformin.

order to alleviate symptoms or slow progression of their disease. Many studies, however, indicate that adherence to medications prescribed for a diagnosed disease is less than optimal. ${ }^{1-3}$ Far less is known about adherence to medications intended to prevent or delay development of a disease among at-risk individuals.

Primary prevention of type 2 diabetes requires long-term adherence to proven lifestyle or medication interventions. Metformin 
has proven safety, efficacy, and tolerability from the Diabetes Prevention Program (DPP $)^{4}$ and the Diabetes Prevention Program Outcomes Study (DPPOS). ${ }^{5}$ It has been recommended in recent standards of medical care for diabetes prevention; ${ }^{6}$ despite this, providers rarely prescribe metformin for diabetes prevention. ${ }^{78}$ While issues related to metformin use for diabetes prevention are multifactorial and at several levels, improved understanding of patient-level factors associated with metformin adherence over time is essential to expanding its use as a preventive medication.

During the first 3 years of DPP, cumulative metformin adherence (defined as the proportion of visits at which participants took $>80 \%$ of prescribed metformin doses) was $71 \%$. While patient-reported barriers were associated with decreased odds of adherence, the use of adherence strategies was associated with increased odds of adherence. Importantly, participants who were adherent to metformin had approximately $25 \%$ reduced risk of developing diabetes compared with those who were not adherent. $^{9}$

This report describes long-term metformin adherence during DPPOS from 2002 through 2013. We examined sociodemographic predictors of adherence over 11 years of follow-up and, based on earlier DPP findings, ${ }^{9}$ we hypothesized a significant association between patientreported barriers and non-adherence. Among those who reported problems with taking metformin, we expected planned strategies to be associated with improved adherence. Based on prior evidence from diverse chronic health conditions, including diabetes, we also hypothesized that poorer physical or mental health and more symptoms of depression or anxiety ${ }^{10-12}$ would be associated with metformin non-adherence. Early non-adherence in DPP and a higher number of concomitant medications were expected to be associated with lower metformin adherence over the follow-up. We explored evidence for cumulative effects of the number of adherence barriers and strategies, as well as effects of specific barrier and strategy types. In addition, the relationship between long-term metformin adherence and time to development of type 2 diabetes was assessed.

\section{RESEARCH DESIGN AND METHODS}

The DPP was a randomized clinical trial testing interventions to prevent or delay the development of type 2 diabetes mellitus among high-risk individuals. ${ }^{4}$ Twentyseven US clinical centers recruited 3234 participants; approximately $45 \%$ were members of ethnic or racial minority groups and $20 \%$ were $\geq 60$ years old. The eligibility criteria included $\geq 25$ years of age, body mass index $\geq 24 \mathrm{~kg} / \mathrm{m}^{2} \quad\left(\geq 22 \mathrm{~kg} / \mathrm{m}^{2}\right.$ in Asian-Americans $)$, fasting plasma glucose (FPG) levels of between 95 and $125 \mathrm{mg} /$ $\mathrm{dL}$ and impaired glucose tolerance (2hours $75 \mathrm{~g}$ postload glucose of $140-199 \mathrm{mg} / \mathrm{dL})$. Eligible participants received standard advice on healthy diet and physical activity and were randomly assigned to intensive lifestyle intervention, metformin $850 \mathrm{mg}$ two times a day or placebo.

\section{Subjects}

This report focuses on DPPOS follow-up data from the metformin group for visits between September 2002 and December 2013. At the end of the main DPP trial in 2001 (mean follow-up 2.8 years), each medication participant had a 1-week to 2-week drug washout period after which eligible metformin participants were offered open-label study metformin. ${ }^{13}$ During this 13-month "bridge" period between DPP and the DPPOS protocols, all study participants were offered a group-administered lifestyle intervention and were invited to enroll in DPPOS for twice-yearly visits. ${ }^{14}$ Participants eligible to continue taking metformin (including those with a diabetes diagnosis, but a FPG $<140 \mathrm{mg} / \mathrm{dL}$ during DPP or an HbAlc $<7 \%$ during DPPOS) continued to receive openlabel study metformin (see figure 1). Written informed consent was obtained from all participants before taking part; the study was approved by each of the 27 clinical centers' and the data coordinating center's institutional review boards (see online DPP Research Group institutions; Approval for GWU Biostatistics Center \#040637). ClinicalTrials.gov registration numbers: NCT00038727, NCT00004992.

\section{Intervention}

The intervention for study-supplied metformin adherence in DPPOS was less intensive than during the DPP and included a brief structured interview at semiannual visits. Participants also received mailed reminder cards intermittently containing motivational messages to promote adherence. The structured adherence interview (online supplemental figure S1) assessed self-reported barriers to adherence and participants' planned strategies to improve adherence. Only participants who took any amount of study-provided metformin since the last visit, and who endorsed having problems taking their metformin as prescribed, completed this interview. The rationale for restricting the interview to this subgroup was the need to reduce the caseload burden of clinic staff. Case managers could initiate telephone counseling between visits for problem-solving medication-taking behaviors, as feasible for staff and acceptable to participants. Participants received token incentives for taking their metformin (eg, a $\$ 10$ gift card annually). Because of budgetary constraints in this long-term study, metformin was dispensed in two large containers, each accommodating a 3-month supply.

\section{Outcome and covariate measures}

Participants were categorized as having 'temporarily' discontinued study metformin if they were eligible to restart study metformin at a later date; reasons for 'temporary' discontinuation included behavioral choices, gastrointestinal (GI) problems, and hospitalization. Participants were categorized as having 'permanently' discontinued 


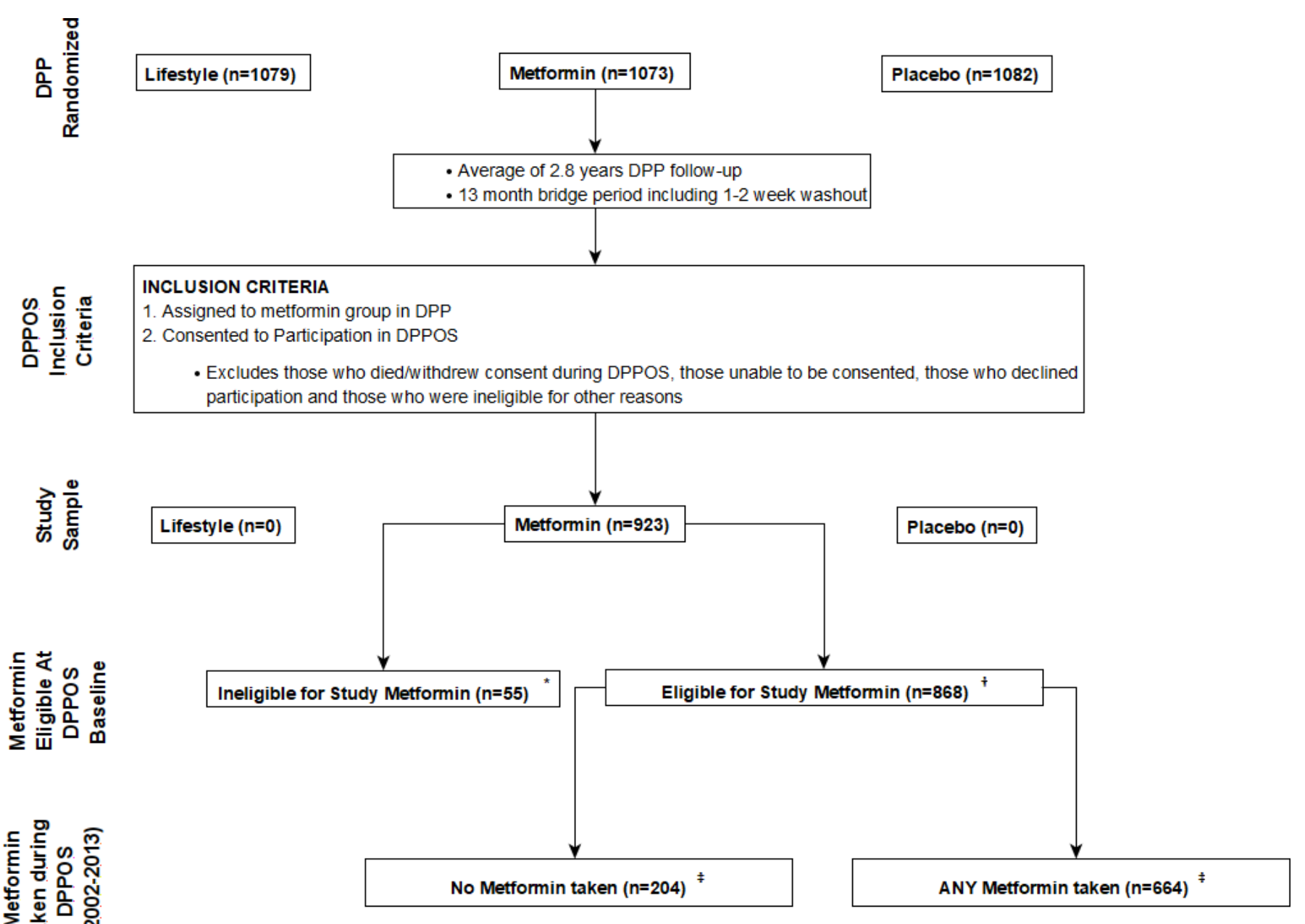

Figure 1 Participant study flow diagram for metformin group. *Permanently discontinued from metformin during the DPP either because fasting plasma glucose $\geq 140 \mathrm{mg} / \mathrm{dL}$ or other protocol deviations and thus ineligible to take study metformin. †This includes 216 participants diagnosed with diabetes during the DPP, but with fasting plasma glucose $<140 \mathrm{mg} / \mathrm{dL}$. $\neq$ For those eligible to take study metformin at DPPOS baseline $(n=868), 216(24.9 \%)$ were diagnosed with diabetes during DPP and a further 235 (27.1\%) were diagnosed during DPPOS follow-up. In those who never took any study metformin during DPPOS $(n=204), 59$ (28.9\%) were diagnosed during DPP while a further $48(23.5 \%)$ were diagnosed during DPPOS. In those who took study metformin at one or more DPPOS visits $(n=664), 157(23.6 \%)$ were diagnosed during DPP while a further $187(28.2 \%)$ were diagnosed during DPPOS. DPP, Diabetes Prevention Program; DPPOS, DPP Outcomes Study.

study metformin for protocol-specified safety and health reasons, such as worsening diabetes requiring primary care provider management (eg, HbAlc $\geq 7 \%$ ) and medical contraindications. Data from visits at or after metformin were permanently discontinued are excluded from all analyses. Adherence was characterized throughout DPP and DPPOS by the convention of $\geq 80 \%$ or $<80 \%$ of prescribed dose, a designation often used in clinical studies. ${ }^{15}$

\section{Visit adherence outcome}

Study staff assessed metformin adherence by pill count at semiannual visits. Adherence was summarized as a dichotomous variable (ie, $<80 \%$ vs $\geq 80 \%$ of prescribed metformin pills taken since last visit), Pill counts were done discretely by DPPOS staff. Participants were categorized as nonadherent at visits when they were 'temporarily' discontinued from study metformin; we acknowledge that some participants were making behavioral choices, while others were temporarily discontinued by protocol.

\section{Cumulative adherence outcome}

This summary measure of adherence over 11 years of visits was computed by determining the number of eligible visits at which a participant was adherent (ie, $\geq 80 \%$ of metformin taken $)^{15}$ and dividing that by the total number of eligible visits, representing the proportion of visits with adequate metformin adherence.

\section{Time to diabetes diagnosis outcome}

This analysis sample includes those who were eligible to take study metformin at time of enrollment in DPPOS (see figure 1), but it excludes those who had already developed diabetes during DPP. Time to diabetes is measured from the start of DPPOS until the date of diabetes diagnosis, defined as a study-measured fasting blood glucose $\geq 126 \mathrm{mg} / \mathrm{dL}$ or 
2 hour postchallenge glucose $\geq 200 \mathrm{mg} / \mathrm{dL}$, confirmed with repeat test within 6 weeks of original test.

\section{Measured covariates}

Demographic and psychosocial variables used to predict adherence were selected based on prior adherence research as well as DPP results. For visits at which a participant was actively taking metformin and reported problems taking it as prescribed, information was recorded during the structured interview on barriers to metformin use over the prior 6 months and planned strategies to overcome these barriers during the subsequent 6 months. Participants could decline to strategize with staff. For participant visits where no problems were reported or where participants were "temporarily" off metformin, the interview was not administered. Therefore, analyses for planned strategies were limited to participants who were actively taking metformin and reported problems with adherence.

Over the 11 years of DPPOS, there were annual assessments of the Beck Depression Inventory, ${ }^{16}$ Beck Anxiety Inventory, ${ }^{17}$ and the Medical Outcomes Study Short Form-36 (SF-36) ${ }^{18}$ Concomitant prescription medications were recorded at each visit. In longitudinal analyses SF-36 scores, Beck Depression scores and number of concomitant medications were summarized as averages during follow-up. An adherence outcome from the 3-month DPP visit $^{9}$ was the measure of early metformin adherence.

\section{Statistical analyses}

The visit adherence outcome was analyzed using generalized estimating equation (GEE) models with a binomial distribution and autoregressive covariance structure to account for the correlation in participant's repeated measurements during follow-up. For barriers to adherence, those reporting only one problem taking study metformin were the reference group. Planned strategy analyses were limited to those reporting problems taking metformin; those declining to discuss adherence strategies were the reference group. The time to diabetes outcome was analyzed using Cox Proportional Hazards models among participants who had not developed diabetes by the beginning of DPPOS. Fit for the Cox Models was assessed using Harrell's c-statistic. ${ }^{19}$ The c-index used in a proportional hazards model is interpreted as a probability that among a randomly chosen pair of individuals, the one with longer survival has an estimated event risk (ie, hazard) that is less than or equal to the event risk for the individual with shorter survival. If the c-statistic were 0.50 , this would indicate that the model has no ability to discriminate between individuals with different survival experience. Values above 0.5 indicate increased model discrimination above pure chance, with values near 1 indicating the model has almost perfect discrimination. These models use all follow-up information of participants until they either develop diabetes or they have their last visit. All models are adjusted for demographic variables, psychosocial variables (depression, anxiety, and SF-36 scores), as well as other concomitant medications and early metformin adherence. Except where noted, $\mathrm{p}<0.05$ were considered nominally statistically significant, with no adjustments made for multiple tests. Analyses were conducted using R (V.3.4.1) and SAS (V.9.4) statistical software.

\section{RESULTS}

\section{Study sample}

Of 1073 participants who were randomized to the metformin group in DPP, $923(86 \%)$ enrolled in the DPPOS (figure 1). Of those 923 enrolled, 868 (94\%) were eligible to take study metformin during DPPOS and 664 of those $(76 \%)$ took at least some study metformin at one or more DPPOS visits. The remaining 204 (24\%) took no study metformin during DPPOS follow-up. Primary reasons for not continuing metformin during DPPOS included: participants stopped it during the 1-2 weeks washout period after DPP and did not restart or patientreported GI issues. The primary focus of this report is the 664 participants who took at least some metformin during DPPOS and a subset $(n=478)$ from whom we collected structured interview adherence data.

\section{Predictors of metformin discontinuation}

The distribution of selected DPP and DPPOS characteristics among the 868 participants who were eligible to take study metformin at start of DPPOS were compared for participants who took any metformin $(n=664)$ versus participants who took no metformin $(n=204)$ during the 11-year follow-up (online supplemental table S1). There were several significant differences between groups, including early adherence to metformin in DPP $(\mathrm{p}<0.001)$, higher income at DPP baseline $(\mathrm{p}=0.02)$, and greater median number of concomitant medications during DPPOS $(\mathrm{p}=0.04)$, related to taking any metformin.

\section{Characteristics by cumulative adherence}

Overall, the cumulative adherence in 11 years of DPPOS was $60 \%$. Table 1 summarizes characteristics among the 868 participants eligible to take metformin during DPPOS across four categories of cumulative adherence. Associated with higher adherence to metformin during DPPOS were: early adherence in DPP $(p<0.001)$, lower median depression scores in DPPOS ( $\mathrm{p}=0.001)$, and higher mean SF-36 physical component scores in DPPOS $(p=0.028)$. Pairwise comparisons found that DPPOS depression scores were significantly lower among those who reached the medication adherence threshold for $\geq 80 \%$ of DPPOS visits than for those who reached the threshold for some, but $<80 \%$ of visits. Those who reached the adherence threshold for $\geq 80 \%$ of visits had the highest SF-36 physical component mean scores; other pairwise comparisons were not significant. There were also significant overall differences by race/ethnicity $(\mathrm{p}=0.004)$. Only $31 \%$ of Blacks were adherent at $\geq 80 \%$ of visits compared with whites (47\%) and Hispanics (48\%). Hispanics had the lowest prevalence of taking no study metformin in DPPOS (20\%). Employment status at DPPOS baseline was associated with greater adherence $(\mathrm{p}=0.003)$. 
Table 1 Characteristics of 868 participants eligible to take study metformin ${ }^{*}$ at DPPOS baseline by category of metformin adherence at $\geq 80 \%$ of prescribed dose during DPPOS

\begin{tabular}{|c|c|c|c|c|c|}
\hline & (1) & (2) & (3) & (4) & \\
\hline Adherence category & $\begin{array}{l}\text { No metformin } \\
\text { taken in DPPOS }\end{array}$ & $\begin{array}{l}\text { Adherent at } 0 \% \text { of } \\
\text { DPPOS visits }\end{array}$ & $\begin{array}{l}\text { Adherent at } \\
>0 \%-<80 \% \text { of } \\
\text { DPPOS visits }\end{array}$ & $\begin{array}{l}\text { Adherent at } \geq \mathbf{8 0} \% \\
\text { of DPPOS visits }\end{array}$ & $P$ value \\
\hline Number of participants & 204 & 42 & 247 & 375 & \\
\hline Age at DPPOS baseline (years) & & & & & 0.141 \\
\hline $30-49$ & $62(23.6 \%)$ & $14(5.3 \%)$ & $89(33.8 \%)$ & $98(37.3 \%)$ & \\
\hline $50-64$ & $98(22.1 \%)$ & $19(4.3 \%)$ & $120(27.1 \%)$ & $206(46.5 \%)$ & \\
\hline$\geq 65$ & $43(26.9 \%)$ & $9(5.6 \%)$ & $37(23.1 \%)$ & $71(44.4 \%)$ & \\
\hline Race/ethnicity† & & & & & 0.004 \\
\hline White & 115 (23.3\%) & $14(2.8 \%)$ & $132(26.8 \%)$ & $232(47.1 \%)$ & \\
\hline Black & $44(25.6 \%)$ & $14(8.1 \%)$ & $60(34.9 \%)$ & $54(31.4 \%)$ & \\
\hline Hispanic & $25(19.5 \%)$ & $7(5.5 \%)$ & $35(27.3 \%)$ & $61(47.7 \%)$ & \\
\hline Otherł & $20(26.7 \%)$ & $7(9.3 \%)$ & $20(26.7 \%)$ & $28(37.3 \%)$ & \\
\hline Sex & & & & & 0.290 \\
\hline Male & 69 (24.4\%) & $11(3.9 \%)$ & $71(25.1 \%)$ & $132(46.6 \%)$ & \\
\hline Female & $135(23.1 \%)$ & $31(5.3 \%)$ & $176(30.1 \%)$ & $243(41.5 \%)$ & \\
\hline Marital status at DPP baseline & & & & & 0.386 \\
\hline Married/Living together & 78 (26.2\%) & $16(5.4 \%)$ & 86 (28.9\%) & 118 (39.6\%) & \\
\hline $\begin{array}{l}\text { Single, separated, divorced, or } \\
\text { widowed }\end{array}$ & $126(22.1 \%)$ & $26(4.6 \%)$ & $161(28.2 \%)$ & $257(45.1 \%)$ & \\
\hline Marital status at DPPOS baseline & & & & & 0.145 \\
\hline Married/Living together & $83(26.9 \%)$ & 17 (5.5\%) & 89 (28.9\%) & 119 (38.6\%) & \\
\hline $\begin{array}{l}\text { Single, separated, divorced, or } \\
\text { widowed }\end{array}$ & $120(21.5 \%)$ & $25(4.5 \%)$ & $157(28.1 \%)$ & $256(45.9 \%)$ & \\
\hline Educational status at DPP baseline & & & & & 0.490 \\
\hline$\leq$ High School & $43(20.5 \%)$ & $15(7.1 \%)$ & $60(28.6 \%)$ & $92(43.8 \%)$ & \\
\hline Attended College & $102(24.5 \%)$ & $20(4.8 \%)$ & $116(27.9 \%)$ & $178(42.8 \%)$ & \\
\hline Attended Graduate School & $59(24.4 \%)$ & $7(2.9 \%)$ & $71(29.3 \%)$ & $105(43.4 \%)$ & \\
\hline Educational status at DPPOS baseline & & & & & 0.126 \\
\hline$\leq$ High School & $37(17.8 \%)$ & $15(7.2 \%)$ & $59(28.4 \%)$ & $97(46.6 \%)$ & \\
\hline Attended College & $111(26.4 \%)$ & $19(4.5 \%)$ & $122(29.0 \%)$ & $169(40.1 \%)$ & \\
\hline Attended Graduate School & $55(23.2 \%)$ & $8(3.4 \%)$ & $65(27.4 \%)$ & $109(46.0 \%)$ & \\
\hline Employment at DPP baseline & & & & & 0.682 \\
\hline Full or part time & $153(23.0 \%)$ & $30(4.5 \%)$ & $191(28.7 \%)$ & $292(43.8 \%)$ & \\
\hline Retired & $31(27.2 \%)$ & $6(5.3 \%)$ & 27 (23.7\%) & $50(43.9 \%)$ & \\
\hline Other§ & $20(22.7 \%)$ & $6(6.8 \%)$ & $29(33.0 \%)$ & $33(37.5 \%)$ & \\
\hline Employment status at DPPOS baseline & & & & & 0.003 \\
\hline Full or part time & $134(21.8 \%)$ & $26(4.2 \%)$ & $185(30.1 \%)$ & $269(43.8 \%)$ & \\
\hline Retired & $43(24.9 \%)$ & $12(6.9 \%)$ & $33(19.1 \%)$ & $85(49.1 \%)$ & \\
\hline Other§ & $26(32.9 \%)$ & $4(5.1 \%)$ & $28(35.4 \%)$ & $21(26.6 \%)$ & \\
\hline Income status at DPP baseline & & & & & 0.076 \\
\hline$<\$ 20000$ & $23(22.5 \%)$ & $6(5.9 \%)$ & $27(26.5 \%)$ & $46(45.1 \%)$ & \\
\hline$\$ 20-\$ 75000$ & $130(25.8 \%)$ & $26(5.2 \%)$ & $144(28.6 \%)$ & $203(40.4 \%)$ & \\
\hline$>\$ 75000$ & $31(15.9 \%)$ & $7(3.6 \%)$ & $56(28.7 \%)$ & $101(51.8 \%)$ & \\
\hline Early metformin adherence at 3 months & in $\mathrm{DPP} * *$ & & & & $<0.001$ \\
\hline Non-adherent & $83(34.6 \%)$ & $18(7.5 \%)$ & 73 (30.4\%) & $66(27.5 \%)$ & \\
\hline
\end{tabular}

Continued 
Table 1 Continued

\begin{tabular}{|c|c|c|c|c|c|}
\hline \multirow[b]{2}{*}{ Adherence category } & \multirow{2}{*}{$\begin{array}{l}\text { (1) } \\
\text { No metformin } \\
\text { taken in DPPOS }\end{array}$} & \multirow{2}{*}{$\begin{array}{l}\text { (2) } \\
\text { Adherent at } 0 \% \text { of } \\
\text { DPPOS visits }\end{array}$} & \multirow{2}{*}{$\begin{array}{l}\text { (3) } \\
\text { Adherent at } \\
>0 \%-<80 \% \text { of } \\
\text { DPPOS visits }\end{array}$} & \multirow{2}{*}{$\begin{array}{l}\text { (4) } \\
\begin{array}{l}\text { Adherent at } \geq 80 \% \\
\text { of DPPOS visits }\end{array}\end{array}$} & \multirow[b]{2}{*}{$P$ value } \\
\hline & & & & & \\
\hline Early adherent & $110(18.2 \%)$ & $20(3.3 \%)$ & $169(28.0 \%)$ & $304(50.4 \%)$ & \\
\hline \multicolumn{6}{|l|}{ Mental Component Score of SF-36 } \\
\hline DPP baseline & $54.1 \pm 7.3$ & $53.4 \pm 8.6$ & $53.4 \pm 8.8$ & $54.6 \pm 7.3$ & 0.305 \\
\hline Mean score during DPPOS & $53.9 \pm 7.1$ & $52.3 \pm 7.0$ & $52.9 \pm 6.9$ & $54.1 \pm 6.4$ & 0.109 \\
\hline \multicolumn{6}{|l|}{ Physical Component Score of SF-36 } \\
\hline DPP baseline & $50.4 \pm 7.4$ & $49.4 \pm 7.5$ & $50.0 \pm 7.4$ & $50.5 \pm 6.8$ & 0.746 \\
\hline Mean score during DPPOS†† & $46.4 \pm 8.3$ & $46.5 \pm 8.8$ & $46.6 \pm 8.0$ & $48.2 \pm 7.1$ & 0.028 \\
\hline \multicolumn{6}{|l|}{ Beck Anxiety Score†† } \\
\hline Median DPP baseline & $2.0(0.0,5.0)$ & $3.0(1.0,6.0)$ & $3.0(1.0,6.0)$ & $2.0(0.0,5.0)$ & 0.313 \\
\hline Median score during DPPOS & $3.0(1.1,5.6)$ & $3.9(1.8,5.8)$ & $3.4(1.6,6.1)$ & $2.8(1.2,5.2)$ & 0.060 \\
\hline \multicolumn{6}{|l|}{ Beck Depression Scoreł‡ } \\
\hline Median DPP baseline & $4.0(1.0,7.0)$ & $3.0(1.0,7.0)$ & $4.0(1.0,7.0)$ & $3.0(1.0,6.0)$ & 0.438 \\
\hline Median score during DPPOSף & $3.1(1.5,6.0)$ & $3.8(1.6,7.2)$ & $3.8(2.1,6.6)$ & $2.7(1.1,5.5)$ & 0.001 \\
\hline \multicolumn{6}{|l|}{ Number of concomitant medications } \\
\hline Median DPP baseline & $0.0(0.0,2.0)$ & $0(0.0,1.0)$ & $1.0(0.0,2.0)$ & $1.0(0.0,2.0)$ & 0.063 \\
\hline Median during DPPOS & $2.2(0.9,3.9)$ & $2(1.2,4)$ & $2.6(1.3,4.3)$ & $2.7(1.4,4.3)$ & 0.105 \\
\hline
\end{tabular}

Percentages add to $100 \%$ across each row. Other statistics are mean \pm SD, or median (25th, 75th percentiles).

Significant pairwise tests (Holm adjusted) of column groups for covariates with significant overall p values (ANOVA for continuous variables, $\chi^{2}$ tests for categorical variables).

DPP baseline data are included when DPPOS baseline data are not available and when a comparison to DPPOS mean data is appropriate.

*DPPOS metformin participants who had a fasting plasma glucose $\geq 140 \mathrm{mg} / \mathrm{dL}$ during DPP were ineligible to continue study metformin during DPPOS. They were advised to consult their private physicians for treatment of their diabetes. Participants with a new diagnosis of diabetes during DPPOS are included until they reach an A1c threshold of $7 \%$.

†Significant pairwise comparisons: 2 vs 4 ( $p<0.05)$.

$\neq$ Combined Asian and American Indians due to small cell size.

§Unemployed, homemaker, student, seasonally employed, never worked, and other.

ISignificant pairwise comparisons: 3 vs $4(p<0.05)$.

**Significant pairwise comparisons: 1 vs 3 ( $p<0.05) ; 1$ vs 4 ( $p<0.05) ; 2$ vs 4 ( $p<0.05) ; 3$ vs 4 ( $p<0.05)$.

††Significant pairwise comparisons: No pairwise comparisons significant.

$\ddagger \ddagger T$ he Beck depression and anxiety scores were calculated from the standard 21-question inventory for each.

ANOVA, analysis of variance; DPP, Diabetes Prevention Program; DPPOS, DPP Outcomes Study; SF-36, Short Form-36.

\section{Barriers and strategies to improve adherence}

Among the $72 \%$ (478/664) of participants who reported barriers while taking metformin during DPPOS, the most frequently reported single barrier was forgetting, followed by multiple barriers and GI complaints, with a significant declining trend in these barriers across the 11 years (figure 2A). The most frequently reported planned (ie, prospective) strategies to improve adherence (figure 2B) were: taking metformin with other routine activity strategies (eg, take with other medications twice each day); setting a specific time routine (eg, take at $8 \mathrm{am}$ ); or reminder devices (eg, a 7-day pill box).

\section{Predicting metformin adherence}

Figure 3 shows forest plots summarizing multivariate GEE models predicting adherence to metformin among those who took at least some metformin during 11 years and who reported barriers to taking metformin as prescribed.
Planning one or more strategies was significantly associated with improved adherence; early DPP adherence was marginally significant $(p=0.053)$ as a predictor of higher DPPOS adherence in this model. Odds of adherence were lower for Black participants and for those who reported $>1$ barrier to adherence (figure $3 \mathrm{~A}$ ).

Figure $3 \mathrm{~B}$ presents the same model evaluating specific types of barriers (reference "forgets") and adherence strategies (reference "declined to strategize"). While reporting multiple barriers lowered adherence, reporting GI problems as a single barrier increased adherence (each marginally at $\mathrm{p}=0.05$ ). Each strategy type significantly increased the odds of adherence. Black race remained associated with lower adherence $(\mathrm{p}<0.001)$.

\section{Modeling time to diabetes}

Figure 4 shows a forest plot of HRs for diabetes incidence among the 652 participants who had not developed 


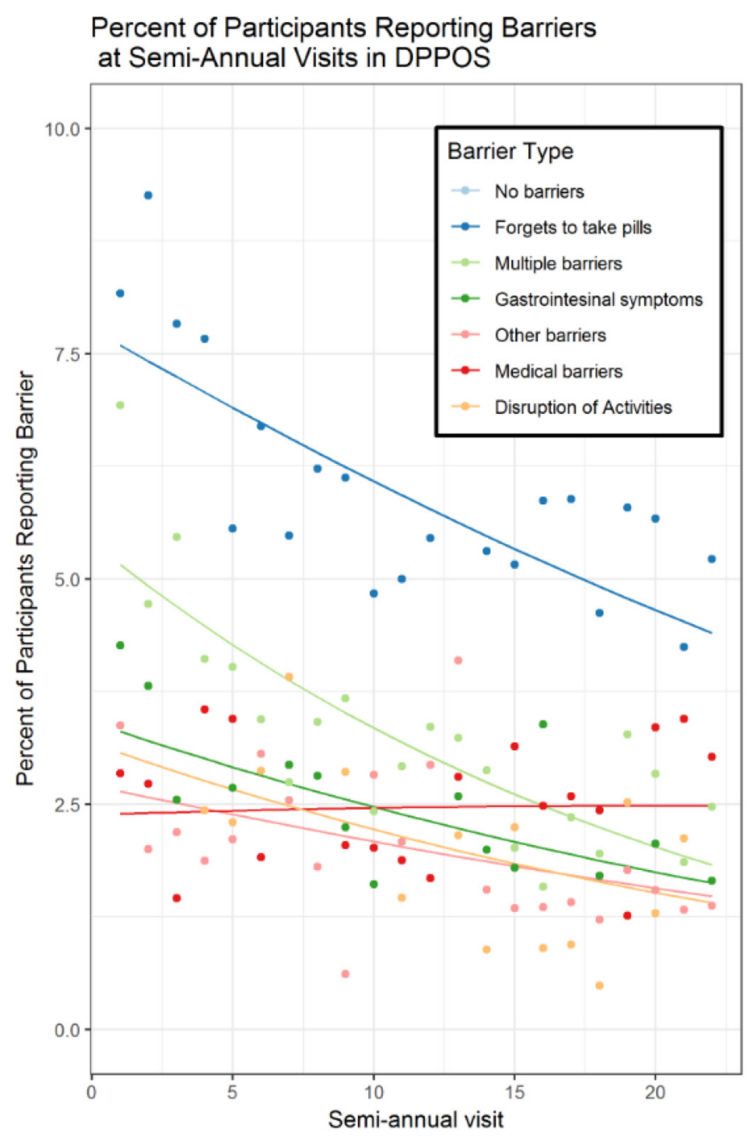

A
Percent of Participants Reporting Strategies at Semi-Annual Visits in DPPOS

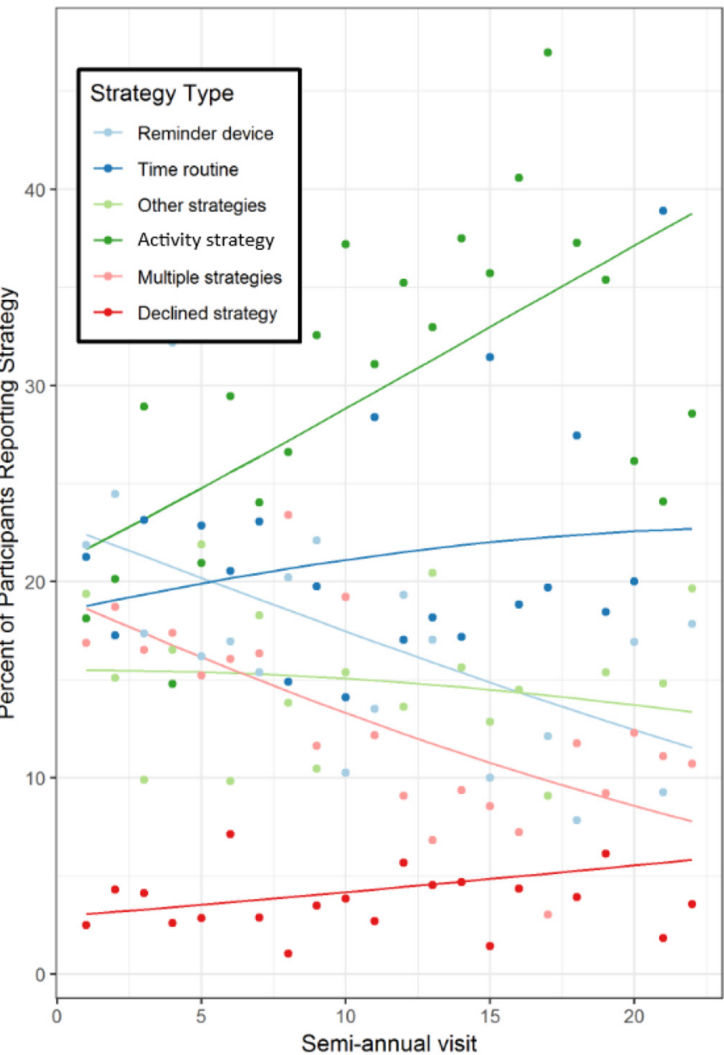

B

Figure 2 Barrier and strategy reporting during DPPOS follow-up ( $n=478)$. Panel A: Per cent of participants who reported specific barriers to adherence to study metformin. Panel B: Per cent of participants who reported specific strategies to improve adherence to study metformin. Dots in the figures represent observed percentages while lines indicate the modeled percentages of participants from a multinomial GEE model with barrier categories and strategy categories as the outcomes. Barrier categories with significant changes over time: no barriers $(p<0.001$; not shown); forgets to take pills $(p<0.01)$; multiple barriers $(p<0.001)$; gastrointestinal symptoms $(p<0.05)$; disruption of activities $(p<0.01)$. Strategy categories with significant changes over time: reminder device $(p<0.001)$; multiple strategies $(p<0.001)$; activity strategy $(p<0.001)$. DPP, Diabetes Prevention Program; DPPOS, DPP Outcomes Study; GEE, generalized estimating equation.

diabetes before the start of DPPOS. The risk of developing diabetes was significantly decreased in those with DPPOS cumulative metformin adherence $\geq 80 \%$ vs $<80 \%$ (HR 0.72; $\mathrm{p}=0.04$ ). The $\mathrm{c}$-index ${ }^{19}$ (a measure of model fit varying between 0.5 and 1 , with higher values indicating better fit,) was used to compare models with and without the two adherence variables (ie, early and cumulative adherence). The c-index (bootstrapped 95\% CI) for the model including both early and cumulative adherence is 0.62 (0.58 to 0.63$)$, and for the model without these adherence measures is 0.60 (0.58 to 0.61 ), giving a small improvement in the predictive capability of the model ( $\Delta$ c-index $=0.02$ ( 0 to 0.04$)$ ) when adherence measures are included. Black race was significantly associated with increased diabetes risk and education with decreased risk in both models.

\section{CONCLUSION}

The DPPOS medication adherence results are a unique contribution to clinical, behavioral literature as they represent 11 years of follow-up, a diverse cohort and an open-label study occurring after the report of positive results for prevention or delay of type 2 diabetes using metformin. A report of long-term safety and tolerability of metformin during DPP and the first 7 years of DPPOS ${ }^{20}$ showed that weight loss was associated with better adherence to metformin and that long-term metformin use was safe and well tolerated. To our knowledge, there are no publications comparable to this report regarding longterm preventive medication adherence, predictors, and clinical outcomes over 11 years. Systematic reviews on interventions to enhance medication adherence note that long-term interventions in chronic disease require more research; few studies report effects on both adherence and clinical outcomes. ${ }^{21}$ In addition, most studies are of therapeutic rather than preventive medications. ${ }^{22}$ This DPPOS adherence report fills important knowledge gaps.

Among those eligible to take study metformin in DPPOS, participants who took at least some metformin 


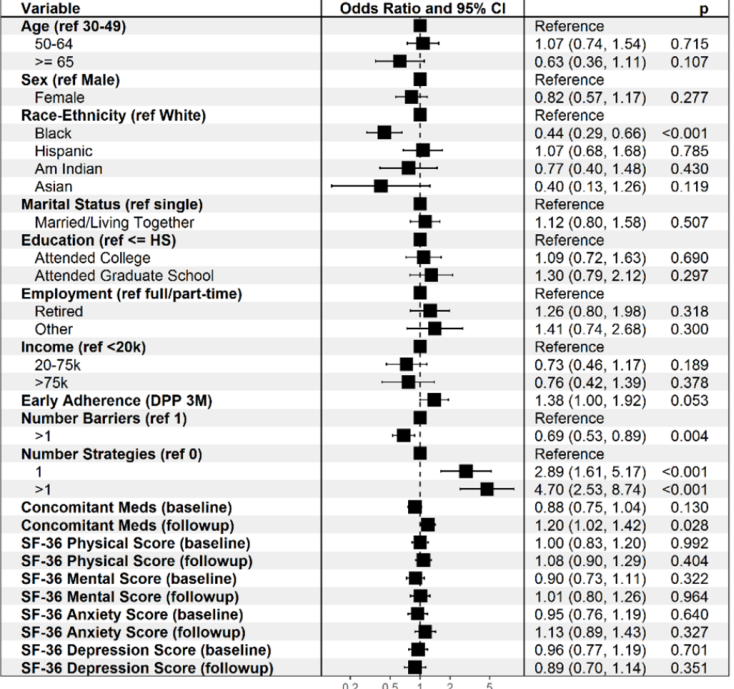

A

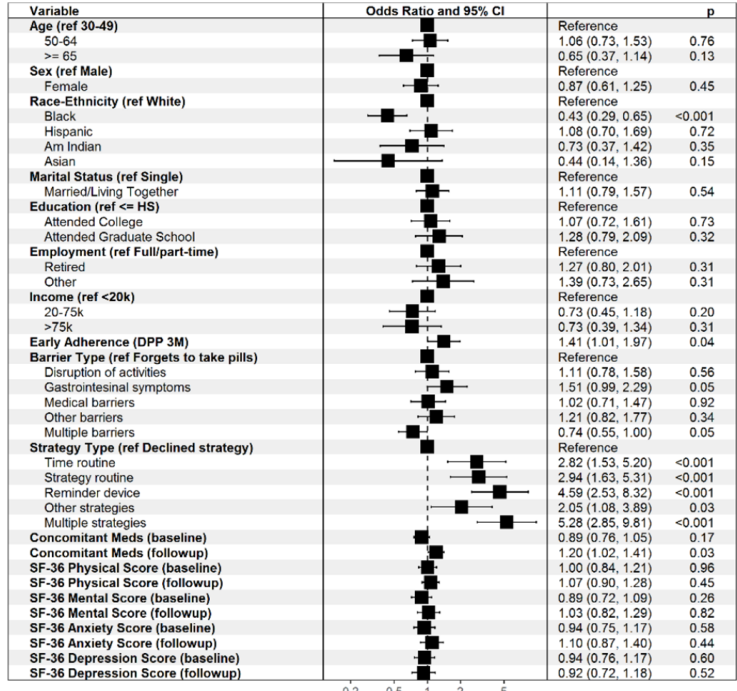

B

Figure 3 ORs for adherence among participants who took study metformin during DPPOS and reported adherence problems at one or more visits $\left(n=478^{\star}\right)$. Panel A: includes assessing number of barriers metformin adherence $(1 \mathrm{vs}>1)$ and number of strategies to promote metformin adherence $(1,>1$ vs 0$)$. Panel B: includes assessing types of barriers to adherence and types of strategies to improve adherence. ORs and Cls were calculated from a GEE model with metformin adherence as the outcome and variables listed in the figure as covariates in a multivariate model. Age, marital status, educational level, employment status, and income level were all measured at DPP baseline. The follow-up SF-36 and concomitant medications variables are averaged over follow-up visits. In these models, barriers and strategies (both number and category) were included as time-varying covariates (ie, the reported values at each participant-visit). Depression scores, anxiety scores, and number of concomitant medications were included in the models as logged values, so estimates of OR for these covariates are expressed per 1-unit log increase. ${ }^{*}$ Only participants who reported problems taking their study metformin at one or more visits were included in this analysis, as only those participants had interview data collected on barriers and strategies to study metformin use. DPP, Diabetes Prevention Program; DPPOS, DPP Outcomes Study; GEE, generalized estimating equation; SF-36, Short Form-36.

were more adherent to metformin early in DPP, had higher income, and took more concomitant medications than those who took no metformin. An important medication adherence finding from the DPP, that early medication adherence predicted adherence at 1 year and 3 years, ${ }^{9}$ was sustained as predictive of cumulative adherence during the 11 years of DPPOS. This effect of early DPP adherence was evident and significant in analyses grouping categories of participants by their level of adherence throughout DPPOS and in analyses that predicted the odds of achieving the adherence threshold over time

Results of analyses (table 1) grouping participants into different levels of adherence over time also supported our hypotheses linking both lower depressive symptoms and a higher physical component score on the SF-36 to better medication adherence. Significant differences among the racial/ethnic categories of participants in DPPOS by categories of adherence (table 1) must be considered with caution because of some small cell sizes. Adherence challenges for Black participants require further exploration. These challenges seemed to have more to do with difficulties achieving consistently high levels of adherence in DPPOS rather than increased risk for stopping metformin. Black participants were at risk for lower adherence across study visits over time, even in multivariate models that controlled for potentially confounding socioeconomic and psychosocial variables.
Those retired at DPPOS baseline were also more likely to have consistently high levels of adherence, perhaps having developed routines for taking metformin.

Compared with the $71 \%$ cumulative metformin adherence during DPP's 3 years, ${ }^{9}$ cumulative adherence during the 11 years of DPPOS was $60 \%$. Although adherence to metformin was a priority for DPPOS staff, some potential explanations for the decline include: aging participants; less personal contact time than during DPP; staff or participant study fatigue and other unmeasured variables.

The majority (ranging from $72 \%$ to $84 \%$ over time) of participants taking any metformin reported no problems/barriers taking metformin as prescribed. For those who did report problems/barriers, the most frequently reported barrier was forgetting to take metformin, similar to reports during the DPP years. ${ }^{9}$ A recent Randomized Clinical Trial (RCT) by Choudhry et $a l^{23}$ assessed the effect on medication adherence of using low-cost reminder devices delivered with no behavioral intervention; there were no significant differences in adherence compared with controls.

Our consistent patterns of adherence over 11 years of DPPOS could be attributed to modest behavioral interventions coupled with optional reminder devices. Most reported barriers significantly declined over time, perhaps the result of problem-solving routines. Medication-taking behaviors may have stabilized for taking or not taking 


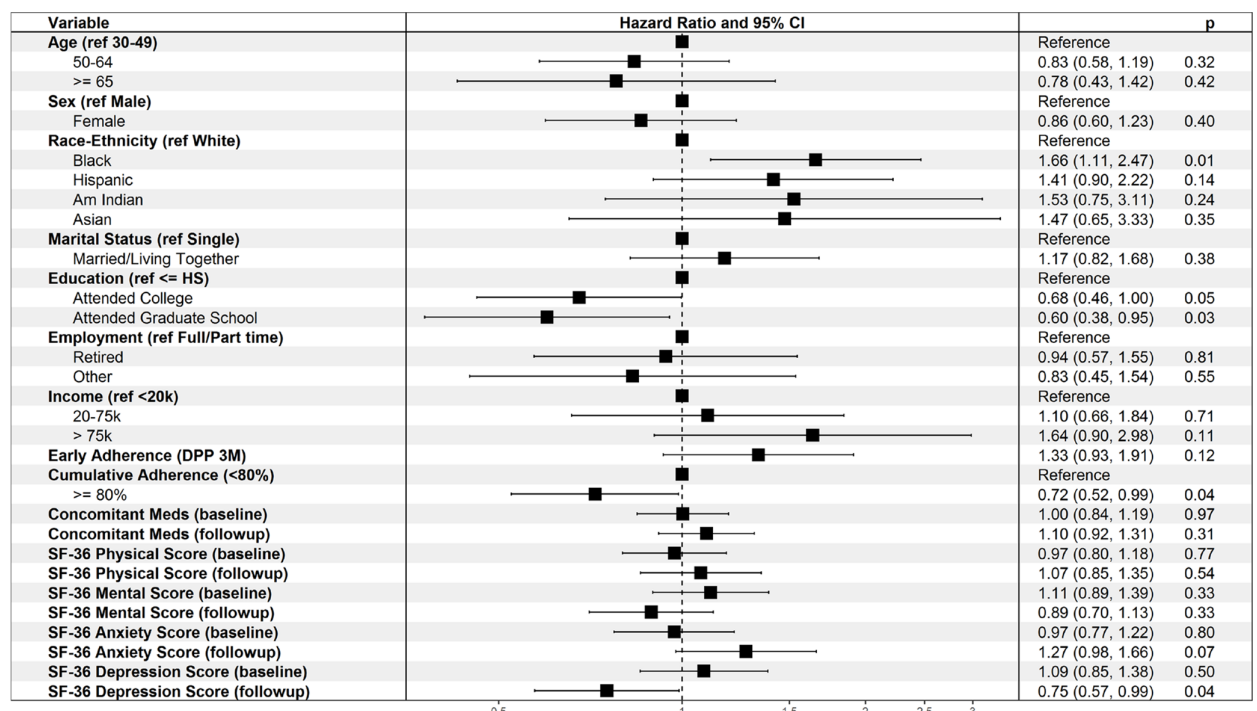

Figure $4 \mathrm{HR}$ for developing diabetes in those who took at least some study metformin during DPPOS ( $\mathrm{n}=652)^{\star}$. HRs and their 95\% Cls from a Cox Proportional Hazards model for time to diabetes measured from DPPOS baseline. All variables listed in the figure are covariates included in a multivariate model. Age, marital status, educational level, employment status, and income level were all measured at DPP baseline. The follow-up SF-36 and number of concomitant medications variables are averages during follow-up. Cumulative metformin adherence is calculated as the cumulative per cent of visits (up to and including each collection time point) at which a participant took $80 \%$ or more of their prescribed study metformin. *Includes only participants whose diagnosis of diabetes, or censoring, occurred on or after August 1, 2002 (the official start date of DPPOS) were included in this analysis. There was a total of 216 participants from this study who developed diabetes prior to the start of DPPOS.

Depression scores, anxiety scores, and number of concomitant medications were included in the models as logged values, so estimates of OR for these covariates are expressed per 1-unit log increase. DPP, Diabetes Prevention Program; DPPOS, DPP Outcomes Study; SF-36, Short Form-36.

metformin. The participants who continued taking metformin were more likely to be adherent at $\geq 80 \%$ of prescribed dose.

Planned strategies to improve adherence to metformin were variable over 11 years of DPPOS, with reminder devices significantly declining in use and activity-related strategies significantly increasing. Comparison of DPP adherence strategy results ${ }^{9}$ and DPPOS results reported here should be done cautiously, as the strategy interview query in DPP was retrospective, that is, strategies used during the last 3 months; the query in DPPOS was prospective, that is, a strategy to improve adherence over the next 6 months.

The models in figure 3 inform predictors of medication adherence in this long-term study. Early adherence in DPP increased the odds of adherence in DPPOS. Black participants had significantly lower odds of adherence during DPPOS, whereas during DPP, Black race was only predictive of lower medication adherence in multivariate analyses for the placebo group. ${ }^{9}$ Early metformin adherence was a strong predictor of adherence at 1 year and 3 years in DPP; this same covariate continued predicting adherence through DPPOS. It is critical to plan adherence interventions from the first dose of a preventive medication, rather than waiting for problems to surface and exposure time lost.

Reporting multiple barriers lowers the odds of adherence and reporting multiple planned strategies raises odds of adherence (figure 3). These results were remarkably similar during DPP for the metformin group and in DPPOS despite the following: DPPOS participants were made aware of DPP positive results for taking metformin to prevent/delay diabetes; the less intensive intervention (eg, reduced frequency of contact) during DPPOS; and limited incentives for adherence during DPPOS.

Survival analyses for diagnosis of diabetes during the DPPOS 11 years (figure 4) showed metformin adherence in DPPOS and higher education decreased the $\mathrm{HR}$, while Black race and mean anxiety score increased it. The significance of DPPOS cumulative adherence depended on adjustment for early adherence in DPP. When early DPP adherence was not controlled, the effect of DPPOS adherence fell slightly below the significance threshold. Therefore, accounting for overlap with early DPP adherence improved, rather than attenuated, the predictive power of DPPOS cumulative adherence for time to diabetes diagnosis. Early DPP adherence was not a significant predictor of time to diabetes in these DPPOS analyses; however, our previously reported results demonstrated a significant association between metformin adherence and time to diabetes within the 2.8 years of DPP. ${ }^{9}$ Taken together, these findings suggest that more proximal metformin adherence is predictive of diabetes risk, irrespective of an earlier history of adherence.

Those who took any metformin during DPPOS $(\mathrm{n}=664)$ reported taking more concomitant medications than those who were taking no metformin (online 
supplemental table S1), a finding opposite to the inverse relationship between complexity of medication regimens and adherence found in earlier reviews. ${ }^{1212425}$ There is some evidence that this relationship depends on drug class. ${ }^{26}$ Some studies in type 2 diabetes have found no decrease in adherence linked to poly-pharmacy. ${ }^{27} \mathrm{~A}$ more recent systematic review summarizes inconsistent findings across studies in type 2 diabetes. ${ }^{28}$ We are aware of no robust studies that have investigated this relationship for adherence to preventive medications. Perhaps providers could take advantage of patients' polypharmacy to promote preventive medication adherence by assisting patients to adopt their established routines to include metformin.

Since forgetting was the most frequently cited barrier in both DPP and DPPOS, new ways of meeting this challenge must be implemented for preventive medication adherence in prediabetes, including behavioral interventions begun at initiation. ${ }^{23}$ For long-term adherence, innovative technologies should be explored. Digital reminders on smart phones are an obvious target for implementing a time-oriented routine, ${ }^{29}$ although the feasibility of taking medications may be challenging when a digital cue (alarm, text message) occurs, unless the medication supply is carried by the individual.

Research on long-term medication adherence for primary prevention in chronic diseases is limited. Newby et $a l^{30}$ reported on long-term adherence to prevention therapies in coronary artery disease in 31750 patients. Adherence was suboptimal for aspirin, lipid-lowering agents, and betablockers; they concluded that more attention be focused on understanding and improving long-term adherence. A systematic review of 62 interventions ${ }^{31}$ to improve medication adherence in chronic disease concluded that while reduced out-of-pocket expenses and case management improved medication adherence, there was no evidence for its effect on long-term adherence and outcomes. Our data may fill some gaps regarding long-term preventive medication adherence.

One limitation of this study is that results do not address non-adherence related to the common barrier of refilling prescription medications; study metformin was dispensed to participants every 6 months. A second limitation is that the time burden of the adherence interview was intentionally decreased for feasibility in this long-term study; it was only asked of participants who reported problems taking their study medication. This limits the generalizability of our findings, as we do not have responses from participants who reported no barriers; they may have used effective strategies to overcome barriers. We did, however, report similar data during the DPP. ${ }^{9}$ The pill count measure of medication adherence has sources of error, ${ }^{32}$ and especially in a long-term study where reported as a dichotomous variable. Finally, we chose to define metformin adherence as exposure (ie, actually taking metformin) versus intention to take metformin among participants who temporarily discontinued use for protocol reasons. Thus, estimates of actual metformin adherence are lower in our study compared with studies in which adherence is primarily defined in behavioral terms (eg, participants who stop study medication for protocol reasons being classified as adherent). We believe each limitation is more than balanced by the strengths of this long-term adherence study with behavioral and clinical outcomes for a preventive diabetes medication in a diverse, well-described cohort.

Lessons from the DPPOS experience can help develop feasible patient-level interventions to improve adherence to metformin for diabetes prevention in the real world. Clearly, early adherence remains an important goal in both clinical practice and research in order to implement effective interventions. Implementing long-term, effective strategies to promote adherence to a preventive medication is a crucial piece of the ongoing type 2 diabetes prevention challenge. Prescription of metformin for diabetes prevention, however, remains underused at the provider and health system levels; ${ }^{7833}$ thus a multilevel approach is required to prevent or delay diabetes with metformin.

\section{Author affiliations}

${ }^{1}$ Medicine, Albert Einstein College of Medicine, Bronx, New York, USA

${ }^{2}$ Ferkauf Graduate School of Psychology, Yeshiva University, New York, New York, USA

${ }^{3}$ The Biostatistics Center, The George Washington University Milken Institute of Public Health, Washington, District of Columbia, USA

${ }^{4}$ Division of Endocrinology, Diabetes \& Metabolism General Clinical Research Center, The University of Tennessee Health Science Center, Memphis, Tennessee, USA

${ }^{5}$ Medicine, University of Chicago, Chicago, Illinois, USA

${ }^{6}$ Medicine, University of Texas Health Sciences Center at San Antonio, San Antonio, Texas, USA

${ }^{7}$ Medicine, University of California at Los Angeles, Los Angeles, California, USA

Acknowledgements The Research Group gratefully acknowledges the commitment and dedication of the participants of the DPP and DPPOS.

Collaborators Please see list of collaborators in supplemental materials. DPP Research Group.

Contributors EAW contributed to the design, interpretation of data, supervision and management of the research, writing, and critical review of the manuscript. JSG contributed to the interpretation of data, statistical analysis plan, writing, and critical review of the manuscript. SD-J contributed to the interpretation of data, writing, and critical review of the manuscript. MJM, MGM and ST contributed to the acquisition of data and critical review of the manuscript. SLE contributed to the design, interpretation of data, supervision and management of the research, statistical analysis, writing, and critical review of the manuscript. MTT contributed to the interpretation of data, management of the research, statistical analysis, writing, and critical review of the manuscript. MTT is the guarantor of this work and, as such, had full access to all the data in the study and takes responsibility for the integrity of the data and the accuracy of the data analysis.

Funding Partial support of EAW and JSG from NIH grant P30 DK 111022. During the DPP and DPPOS, the National Institute of Diabetes and Digestive and Kidney Diseases (NIDDK) of the National Institutes of Health provided funding to the clinical centers and the Coordinating Center for the design and conduct of the study, and collection, management, analysis, and interpretation of the data (U01 DK048489). The Southwestern American Indian Centers were supported directly by the NIDDK, including its Intramural Research Program, and the Indian Health Service. The General Clinical Research Center Program, National Center for Research Resources, and the Department of Veterans Affairs supported data collection at many of the clinical centers. Funding was also provided by the National Institute of Child Health and Human Development, the National Institute on Aging, the National Eye Institute, the National Heart Lung and Blood Institute, the National Cancer Institute, the Office of Research on Women's Health, the National Institute on Minority Health and Health Disparities, the Centers for Disease Control and Prevention, and the American Diabetes Association. Merck KGaA provides medication for DPPOS. 
DPP/DPPOS have also received donated materials from Bristol-Myers Squibb, Parke-Davis, and LifeScan Inc. This research was also supported, in part, by the intramural research program of the NIDDK. LifeScan Inc., Health 0 Meter, Hoechst Marion Roussel, Inc., Merck-Medco Managed Care, Inc., Merck and Co., Nike Sports Marketing, Slim Fast Foods Co., and Quaker Oats Co. donated materials, equipment, or medicines for concomitant conditions. McKesson BioServices Corp., Matthews Media Group, Inc., and the Henry M. Jackson Foundation provided support services under subcontract with the Coordinating Center. The sponsor of this study was represented on the Steering Committee and played a part in study design, how the study was done, and publication. The funding agency was not represented on the writing group, although all members of the Steering Committee had input into the report's contents. All authors in the writing group had access to all data. A complete list of Centers, investigators, and staff can be found in the online supplemental file.

Disclaimer The opinions expressed are those of the investigators and do not necessarily reflect the views of the funding agencies. The funders had no role in study design, data collection and analysis, decision to publish, or preparation of the manuscript.

Competing interests None declared.

Patient consent for publication Not required.

Provenance and peer review Not commissioned; externally peer reviewed.

Data availability statement Data may be obtained from a third party and are not publicly available. In accordance with the NIH Public Access Policy, we continue to provide all manuscripts to PubMed Central including this manuscript. DPP/DPPOS has provided the protocols and lifestyle and medication intervention manuals to the public through its public website (https://www.dppos.org). The DPPOS abides by the NIDDK data sharing policy and implementation guidance as required by the NIH/NIDDK (https://www.niddkrepository.org/studies/dppos/).

Open access This is an open access article distributed in accordance with the Creative Commons Attribution Non Commercial (CC BY-NC 4.0) license, which permits others to distribute, remix, adapt, build upon this work non-commercially, and license their derivative works on different terms, provided the original work is properly cited, appropriate credit is given, any changes made indicated, and the use is non-commercial. See: http://creativecommons.org/licenses/by-nc/4.0/.

ORCID iDs

Elizabeth Arquin Walker http://orcid.org/0000-0002-6328-7673

Samuel Dagogo-Jack http://orcid.org/0000-0001-5318-9677

Sharon L Edelstein http://orcid.org/0000-0001-8457-4404

\section{REFERENCES}

1 DiMatteo MR. Variations in patients' adherence to medical recommendations: a quantitative review of 50 years of research. Med Care 2004;42:200-9.

2 Iglay K, Cartier SE, Rosen VM, et al. Meta-Analysis of studies examining medication adherence, persistence, and discontinuation of oral antihyperglycemic agents in type 2 diabetes. Curr Med Res Opin 2015;31:1283-96.

3 Polonsky WH, Henry RR. Poor medication adherence in type 2 diabetes: recognizing the scope of the problem and its key contributors. Patient Prefer Adherence 2016;10:1299-307.

4 Knowler WC, Barrett-Connor E, Fowler SE, et al. Reduction in the incidence of type 2 diabetes with lifestyle intervention or metformin. N Engl J Med 2002;346:393-403.

5 Diabetes Prevention Program Research Group. Long-Term effects of lifestyle intervention or metformin on diabetes development and microvascular complications over 15-year follow-up: the diabetes prevention program outcomes study. Lancet Diabetes Endocrinol 2015;3:866-75.

6 American Diabetes Association. 5. Prevention or Delay of Type 2 Diabetes: Standards of Medical Care in Diabetes-2018. Diabetes Care 2018;41:S51-4.

7 Moin T, Li J, Duru OK, et al. Metformin prescription for insured adults with prediabetes from 2010 to 2012: a retrospective cohort study. Ann Intern Med 2015;162:542-8.

8 Schmittdiel JA, Adams SR, Segal J, et al. Novel use and utility of integrated electronic health records to assess rates of prediabetes recognition and treatment: brief report from an integrated electronic health records pilot study. Diabetes Care 2014;37:565-8.

9 Walker EA, Molitch M, Kramer MK, et al. Adherence to preventive medications: predictors and outcomes in the diabetes prevention program. Diabetes Care 2006;29:1997-2002.
10 DiMatteo MR, Lepper HS, Croghan TW. Depression is a risk factor for noncompliance with medical treatment: meta-analysis of the effects of anxiety and depression on patient adherence. Arch Intern Med 2000;160:2101-7.

11 Gonzalez JS, Peyrot M, McCarl LA, et al. Depression and diabetes treatment nonadherence: a meta-analysis. Diabetes Care 2008;31:2398-403.

12 Ryan R, Santesso N, Lowe D, et al. Interventions to improve safe and effective medicines use by consumers: an overview of systematic reviews. Cochrane Database Syst Rev 2014;4:CD007768.

13 Diabetes Prevention Program Research Group. Effects of withdrawal from metformin on the development of diabetes in the diabetes prevention program. Diabetes Care 2003;26:977-80.

14 Diabetes Prevention Program Research Group, Knowler WC, Fowler SE, et al. 10-Year follow-up of diabetes incidence and weight loss in the diabetes prevention program outcomes study. Lancet 2009;374:1677-86.

15 Ho PM, Rumsfeld JS, Masoudi FA, et al. Effect of medication nonadherence on hospitalization and mortality among patients with diabetes mellitus. Arch Intern Med 2006;166:1836-41.

16 Beck A, Steer R. Manual of the Beck depression inventory. San Antonio: Psychological Corporation, 1993.

17 Beck AT, Epstein N, Brown G, et al. Beck anxiety inventory 1988;2.

18 McHorney CA, Ware JE, Raczek AE. The mos 36-Item short-form health survey (SF-36): II. psychometric and clinical tests of validity in measuring physical and mental health constructs. Med Care 1993;31:247-63.

19 Harrell FE, Lee KL, Mark DB. Multivariable prognostic models: issues in developing models, evaluating assumptions and adequacy, and measuring and reducing errors. Stat Med 1996;15:361-87.

20 Diabetes Prevention Program Research Group. Long-Term safety, tolerability, and weight loss associated with metformin in the diabetes prevention program outcomes study. Diabetes Care 2012;35:731-7.

21 Nieuwlaat R, Wilczynski N, Navarro T, et al. Interventions for enhancing medication adherence. Cochrane Database Syst Rev 2014;11)::CD000011.

22 Horne R, Chapman SCE, Parham R, et al. Understanding patients' adherence-related beliefs about medicines prescribed for longterm conditions: a meta-analytic review of the Necessity-Concerns framework. PLoS One 2013;8:e80633.

23 Choudhry NK, Krumme AA, Ercole PM, et al. Effect of reminder devices on medication adherence: the remind randomized clinical trial. JAMA Intern Med 2017;177:624-31.

24 Claxton AJ, Cramer J, Pierce C. A systematic review of the associations between dose regimens and medication compliance. Clin Ther 2001;23:1296-310.

25 Choudhry NK, Fischer MA, Avorn J, et al. The implications of therapeutic complexity on adherence to cardiovascular medications. Arch Intern Med 2011;171:814-22.

26 van Bruggen R, Gorter K, Stolk RP, et al. Refill adherence and polypharmacy among patients with type 2 diabetes in general practice. Pharmacoepidemiol Drug Saf 2009;18:983-91.

27 Grant RW, Devita NG, Singer DE, et al. Polypharmacy and medication adherence in patients with type 2 diabetes. Diabetes Care 2003;26:1408-12.

28 Krass I, Schieback P, Dhippayom T. Adherence to diabetes medication: a systematic review. Diabet Med 2015;32:725-37.

29 Thakkar J, Kurup R, Laba T-L, et al. Mobile telephone text messaging for medication adherence in chronic disease: a metaanalysis. JAMA Intern Med 2016;176:340-9.

30 Newby LK, LaPointe NMA, Chen AY, et al. Long-Term adherence to evidence-based secondary prevention therapies in coronary artery disease. Circulation 2006;113:203-12.

31 Viswanathan M, Golin CE, Jones CD, et al. Interventions to improve adherence to self-administered medications for chronic diseases in the United States: a systematic review. Ann Intern Med 2012;157:785-95.

32 Dunbar-Jacob J, Sereika SM, Houze M, et al. Accuracy of measures of medication adherence in a cholesterol-lowering regimen. West $J$ Nurs Res 2012;34:578-97.

33 Cefalu WT, Buse JB, Tuomilehto J, et al. Update and next steps for real-world translation of interventions for type 2 diabetes prevention: reflections from a diabetes care editors' expert forum. Diabetes Care 2016;39:1186-201.

34 Saydah SH. Medication Use and Self-Care in Persons with Diabetes: Chapter 39 in Diabetes in America. In: Cowie C, Casagrande S, Menke A, eds. Nih PUB no. Bethesda: National Institutes of Health, 2018: $14.17-1468$ 